\title{
Continuous intake of Trehalose induces white adipose tissue Browning and Enhances energy metabolism
}

Chikako Arai", Norie Arai, Shigeyuki Arai, Chiyo Yoshizane, Satomi Miyata, Akiko Mizote, Aki Suyama, Shin Endo, Toshio Ariyasu, Hitoshi Mitsuzumi and Shimpei Ushio

\begin{abstract}
Background: Trehalose is well known as a functional disaccharide with anti-metabolic activities such as suppression of adipocyte hypertrophy in mice and alleviation of impaired glucose tolerance in humans. Recently, a new type of adipocyte beige cells, involved in so-called white adipocyte tissue (WAT) browning, has received much attention as a target for adaptive thermogenesis. To clarify the relationship between adipocyte hypertrophy suppression and beige cells involved in thermogenesis, we examined the effect of trehalose on the changes in beige adipocytes in mice under normal dietary conditions.
\end{abstract}

Methods: Mice fed a normal diet were administered water containing 0.3\% (W/) trehalose for 16 weeks, 0.3\% (W/ $\checkmark)$ maltose, or water without saccharide (controls). Body temperature and non-fasting blood glucose levels were measured every 3 weeks. After 16 weeks of these treatments, mesenteric and inguinal adipose tissues were collected for measuring adipocyte size, counting the number of UCP1 positive cells by image analysis, and preparing mRNA to analyze beige adipocyte-related gene expression.

Results: Mice administered a continuous intake of trehalose exhibited a thermogenic ability as represented by an increase in rectal temperature, which was maintained at a relatively high level from 3 to 9 weeks and was significantly higher at 15 weeks in comparison with that of the maltose group. In addition to the reduced hypertrophy of mesenteric and inguinal adipose tissues, the trehalose group showed a significant increase in the rates of beige adipocytes in each WAT in comparison with those of the maltose and the water groups. Interestingly, a negative correlation was found between the mean cell sizes of adipocytes and the rates of beige adipocytes in the WAT. Furthermore, real-time PCR showed that the expression of Cidea and Ucp1 mRNAs, which are markers for beige adipocytes in the inguinal adipose tissue, increased in the trehalose group.

Conclusions: Continuous administration of trehalose to mice fed a normal diet induced WAT browning accompanied by suppression of white adipocyte hypertrophy, elevated body temperature and decreased blood glucose levels, which resulted in enhancement of energy metabolism. Therefore, we propose trehalose as a new type of thermogenic dietary component to prevent obesity by promoting WAT browning.

Keywords: Trehalose, Adipocyte size, Beige adipocyte, UCP1, Temperature

* Correspondence: chikako.arai@hb.nagase.co.jp

HAYASHIBARA CO. LTD, 675-1 Fujisaki, Naka-ku, Okayama 702-8006, Japan

(C) The Author(s). 2019 Open Access This article is distributed under the terms of the Creative Commons Attribution 4.0 International License (http://creativecommons.org/licenses/by/4.0/), which permits unrestricted use, distribution, and reproduction in any medium, provided you give appropriate credit to the original author(s) and the source, provide a link to the Creative Commons license, and indicate if changes were made. The Creative Commons Public Domain Dedication waiver (http://creativecommons.org/publicdomain/zero/1.0/) applies to the data made available in this article, unless otherwise stated. 


\section{Background}

The continuous worldwide increase in obesity is a serious health problem [1]. One promising approach to tackling the obesity problem is to understand the control mechanisms of adipocytes. Adipose tissue comprises white adipocyte tissue (WAT) and brown adipocyte tissue (BAT), both of which are related to energy homeostasis. When excessive energy is ingested, WAT stores energy in the form of triglycerides in unilocular white adipocytes, and it secretes many adipokines such as tumor necrosis factor- $\alpha$ (TNF- $\alpha$ ), interleukin-6 (IL-6), and plasminogen activator inhibitor-1 (PAI-1), which induce insulin resistance $[1,2]$. In contrast, multiple local BAT dissipates excess energy through thermogenesis, based on its high mitochondrial content and the expression of uncoupling protein 1 (UCP1) [3, 4]. Induction of thermogenesis in BAT is a promising method for the treatment of obesity $[5,6]$. However, the amount of BAT in adults is quite small $[3,7]$. Recently, brown-like adipocytes called beige adipocytes were found in WAT, and they expressed UCP1 to dissipate energy, similar to brown adipocytes [8-10]. The inducing phenomenon of beige adipocytes in WAT is called "browning". Induction of the browning increases energy consumption and could help to improve metabolic disorders [5, 6, 9].

Trehalose, a non-reducing disaccharide consisting of two D-glucose residues, has been associated with various biological effects, such as inhibition of bone resorption [11], suppression of inflammatory responses [12, 13], alleviation of Huntington's disease [14], and inhibition of atherosclerotic plaque formation [15]. Recently, we demonstrated that trehalose has anti-metabolic abilities such as lowering insulin secretion $[16,17]$. When obese mice were fed a high fat diet (HFD) and administered drinking water containing $0.3 \%(\mathrm{~W} / \mathrm{V})$ trehalose from 8 to 15 weeks, adipocyte hypertrophy was suppressed, insulin resistance was alleviated, glucose tolerance was improved [18, 19], and hypertrophy of the Langerhans islands was prevented [20]. Since glucose, maltose, highfructose corn syrup, and fructose did not have such effects $[18,20]$, these effects were thought to be specific for trehalose.

Based on the results of an oral glucose tolerance test administered to mice fed a HFD and given drinking water containing trehalose for a long period, we demonstrated that one of the mechanisms of adipocyte hypertrophy inhibition is lowered insulin secretion, because insulin increases the accumulation of fat [18, 19, 21]. However, the other mechanisms by which trehalose inhibits adipocyte hypertrophy are not yet known.

On the other hand, in human studies, subjects who ingested $10 \mathrm{~g} /$ day of trehalose for 12 weeks showed improvement in glucose tolerance under normal dietary conditions [22]. Although this has not been evaluated yet, trehalose is considered to be able to suppress adipocytes even under normal conditions. For anti-obesity, it is necessary to suppress the transition from healthy to obesity as well as to improve from the obese state. Therefore, regulation of adipocytes in normal conditions, especially the increase of beige adipocytes, is important for anti-obesity. However, the effect of trehalose in normal state on adipocytes has not been clarified yet. Therefore, the purpose of this study is to examine the effects of trehalose on beige and brown adipocytes under the normal feeding conditions. Besides the measurement of adipocyte size in both mesenteric and inguinal adipose tissues, we examine the rates of UCP1 positive cells to analyze the induction of beige adipocytes.

\section{Materials and methods \\ Animals}

Ten-week-old female C57BL/6 J mice were obtained from CLEA Japan, Inc. (Tokyo, Japan) and fed a standard diet (CE-2; CLEA Japan, Inc.) and water ad libitum for a week. The mice were kept in a temperaturecontrolled room with a 12-h light cycle. This study was approved by the Laboratory Animal Care Committee of the Hayashibara Co., Ltd. (Okayama, Japan), and all animal experiments were conducted in accordance with the Guidelines for Care and Use of Laboratory Animals of the Hayashibara Co., Ltd.

\section{Test substances}

TREHA $^{\mathrm{Tm}}$ (Hayashibara Co. Ltd.) was used as the source of trehalose. It contains more than $98.0 \%$ trehalose dihydrate. SUNMALT ${ }^{\mathrm{Tm}}$-S (Hayashibara Co. Ltd.) was used as the source of maltose, containing at least $92 \%$ maltose monohydrate.

\section{Study design}

The experimental protocol is shown in Fig. 1. A total of 48 mice were used in this study. After a week of acclimatization, the 11-week-old mice were randomly divided into 3 groups, which were matched for body weight. Detailed grouping is shown in Fig. 1. Two groups of mice were fed a commercial normal diet (CE2 ) and then administered either $0.3 \%(\mathrm{~W} / \mathrm{V})$ trehalose or $0.3 \%(\mathrm{~W} / \mathrm{V})$ maltose in drinking water ad libitum, respectively ( $n=16$, for each group). As the experimental control, another group of mice received a normal diet and water alone $(n=16)$. Four mice were grouped per cage; each cage had to contain animals of a single group. Intake of food and water, which were replaced every other day, was monitored, and body weights were recorded weekly throughout the experiment. The skin temperature of the interscapular region was measured by an emission thermometer (\#53006, Yokogawa Test \& Measurement Corporation, Tokyo, Japan) every 3 weeks, 


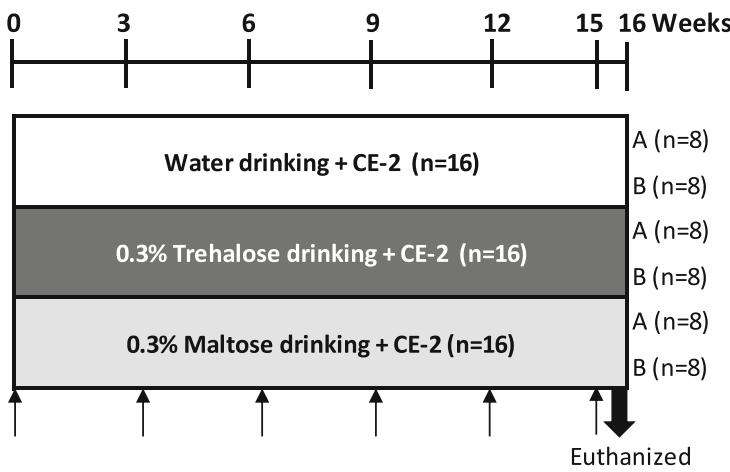

- Divided into 3 groups that were matched for body weight Furthermore, each group was divided into two groups for tissue evaluation(groupA) and genetic analysis(groupB)

- Starting trehalose administration : 0

At $0,3,6,9,12$ and 15 weeks, body temperature and blood glucose were measured

Body and organ weights, food intake, water consumption, body temperature and blood glucose were measured in 16 mice (groupA + groupB).

Adipocyte size, the proportion of UCP1 positive cells, fat degeneration score and serum FGF-21 were analysed in 8 mice (group A).

Beige adipocyte related gene expression rates were analysed in another 8 mice (group B).

Fig. 1 Experimental protocol. After a week of acclimation, all mice were divided into 3 groups matched by body weight and then administered $0.3 \%$ (WN) trehalose or $0.3 \%(W N)$ maltose in drinking water, or water alone. Interscapular and rectal temperature and non-fasting blood glucose were measured every 3 weeks ( $n=16$, group A + group B). The mice were euthanized at 16 weeks. Histological examination and analysis of adipose tissues and measurement of serum FGF-21 protein were performed using samples from 8 mice (group A). Analysis of gene expression in adipose tissues were performed another 8 mice (group B). FGF-21: fibroblast growth factor-21

the rectal temperature was measured using a rectal thermometer (KN-91-AD1687-M, Natsume Seisakusho Co., Ltd., Tokyo, Japan), and non-fasting blood glucose levels were measured with a simple blood glucose meter (Accu-Chek ${ }^{\ominus}$ Aviva Nano meter, Roche Diabetes Care Inc., Indianapolis, IN). After 16 weeks of treatment, the mice were euthanized under pentobarbital anesthesia. Adipose tissue was weighed, and blood samples were collected from the abdominal vena cava to measure fibroblast growth factor-21 (FGF-21). The FGF-21 was measured using an enzyme-linked immunosorbent assay kit (Mouse/Rat FGF-21 Immunoassay, R\&D Systems Inc., Minneapolis, MN).

\section{Calculation of energy intake}

The energy densities of the normal diet and of the drinking water containing $0.3 \%(\mathrm{~W} / \mathrm{V})$ trehalose or $0.3 \%(\mathrm{~W} / \mathrm{V})$ maltose were $14.2 \mathrm{~kJ}, 0.05 \mathrm{~kJ}$, and $0.05 \mathrm{~kJ}$ per gram, respectively. Based on these data, the mean energy intake per mouse in each group was calculated using the following formulas:

Energy intake (kJ/mouse/day)
Mice consuming a normal diet and $0.3 \%(\mathrm{~W} / \mathrm{V})$ trehalose or $0.3 \%(\mathrm{~W} / \mathrm{V})$ maltose

Food intake $(\mathrm{g}) \times 14.2(\mathrm{~kJ})+$ water intake $(\mathrm{g}) \times 0.05(\mathrm{~kJ})$

Mice consuming a normal diet and water

Food intake $(\mathrm{g}) \times 14.2(\mathrm{~kJ})+$ water intake $(\mathrm{g}) \times 0(\mathrm{~kJ})$

\section{Histological analysis of adipocyte size}

Mesenteric adipose tissue samples and inguinal adipose tissue samples were fixed with $10 \%(\mathrm{~V} / \mathrm{V})$ buffered formalin and embedded in paraffin. Sections were deparaffinized in xylene, stained with hematoxylin and eosin, and then examined using light microscopy. Photographs of the respective adipose tissue sections were taken of 5 random areas per section at $400 \times$ magnification. More than 200 adipocyte sizes were measured by image analysis software (cellSens, Olympus Corporation, Tokyo, Japan).

\section{Rates of UCP1 positive cells in white adipose tissues}

Sections of mesenteric adipose tissue and inguinal adipose tissue were stained with anti-UCP1 antibody (diluted at 1:200, ab23841, Abcam, Cambridge, UK) and then stained with secondary antibody (anti-rabbit diluted 1:2, Dako EnVision ${ }^{\oplus}$ + system-HRP labelled polymer; 
Agilent Technologies, Santa Clara, CA). Photographs were taken of 5 random areas of each adipose tissue sample at $400 \times$ magnification. The number of UCP1 positive cells among the total adipocytes (500-800 cells) was measured by cellSens, and the rates of UCP1 positive cells were calculated.

\section{RNA extraction and quantitative real-time polymerase chain reaction (PCR)}

Each mesenteric and inguinal adipose tissue was homogenized in the presence of QIAzol Lysis Reagent (Qiagen, Hilden, Germany) using a TissueRuptor (Qiagen). The respective homogenates were separated into aqueous and organic phases by the addition of chloroform. According to the respective user manuals, total RNAs from the aqueous phase were isolated using an RNeasy Mini Kit (Qiagen) and DNase (Qiagen). Subsequently, firststrand cDNA was synthesized using SuperScript ${ }^{\text {Tw }}$ VILO $^{\text {mix }}$ Master mix (ThermoFisher Scientific, Waltham, MA). Specific primers for PCR analysis were designed using Primer 3 software (http://primer3.sourceforge.net/) (Table 1). The synthesized cDNA was mixed with SYBR ${ }^{\mathrm{mx}}$ Green Master Mix (ThermoFisher Scientific) and different sets of gene-specific primers. Real-time PCR was performed using a LightCycler 480 system (Roche Diagnostic K.K., Tokyo, Japan). For quantitative purposes, the expression levels of the respective target genes were normalized to the housekeeping gene $18 \mathrm{~S}$ rRNA.

\section{Statistics}

The data is expressed as means \pm standard deviations. A power analysis (G*Power 3.1.9.4, http://www.gpower. hhu.de/) showed that the sample size of 8 mice in each group was suitable for detecting a difference between the 3 experimental groups $(1-\beta=0.80$, effect size $=0.7$, $\alpha=0.05)$. In addition, the calculated $p$ values are described. Statistically significant effects of trehalose were examined using Tukey-Kramer (JMP 9.0: SAS, Cary, NC, USA) in the case of dispersion uniformity and normality. Non-parametric data were analyzed by the SteelDwass or Steel test or Wilcoxon test (JMP 9.0: SAS). A $p$-value less than 0.05 was considered significant.

\section{Results}

Continuous ingestion of trehalose showed no effect on energy intake, body weight, and tissue weight

The energy intakes during the experiment were $48.1 \pm$ $1.8,47.2 \pm 1.8$, and $47.7 \pm 1.6 \mathrm{~kJ}$ per mouse per day in the water, trehalose, and maltose groups, respectively. There was no significant difference in the energy intake between the trehalose and the maltose groups. Among the three groups, there were no significant differences in food and water consumption, and the weights of whole body and each organ $(p<0.05)$ are shown in Figs. 2a to c and Table 2.

\section{Trehalose exhibited thermogenic ability at core body sites}

We first examined the thermogenic ability of trehalose by measuring body temperature in the scapular and the rectal regions. The surface temperature of the interscapular region showed no significant difference between the trehalose and the other two groups. The only observable difference was that the maltose group showed slightly lower temperatures at several points, as shown in Fig. 3a. When we measured the rectal temperature, trehalose appeared to exhibit thermogenic abilities, which were detected as a significantly higher temperature $(p<0.05)$ from 12 to 15 weeks in comparison with those of the maltose and the water groups, respectively. In addition, a tendency towards higher temperature (but not significant) was also observed in the trehalose group from 6 to 9 weeks (Fig. 3b).

\section{Blood glucose}

When non-fasting blood glucose was measured after a light phase of 2 to $3.5 \mathrm{~h}$ at 15 weeks of the experiment, the blood glucose level of the trehalose group tended to be lower than that of the water group $(p=0.09)$, as shown in Fig. 4.

\section{Trehalose suppressed adipocyte hypertrophy in mesenteric and inguinal adipose tissues}

We examined the histology of the WAT in the mesenteric and the inguinal adipose tissues and measured the adipocyte sizes from both WAT using the image software cellSens. Histologically, the WAT in both the

Table 1 Summary of the sequences of gene-specific real-time PCR primers

\begin{tabular}{llll}
\hline Target & GenBank accession No. & Forward $\left(5^{\prime} \rightarrow 3^{\prime}\right)$ & Reverse $\left(5^{\prime} \rightarrow 3^{\prime}\right)$ \\
\hline 18S rRNA & & ACTCAACACGGGAACCTCACC & CCAGACAAATCGCTCCACCAAC \\
Cidea & NM_007702 & ACAGAAATGGACACCGGGTA & TCCTTAACACGGCCTTGAA \\
Ucp1 & NM_009463 & GGGCCCTTGTAAACAACAAA & GAAGCCACAAACCCTTTGAA \\
Prdm16 & NM_027504 & CCAGATGTCAGCCATAGAAACC & TCTTGCCACAGTACCTGCAC \\
Pgc1a & NM_008904 & ATGTGTCGCCTTCTTGCTCT & AGGGAGAATTGCGGTGTGT \\
\hline
\end{tabular}



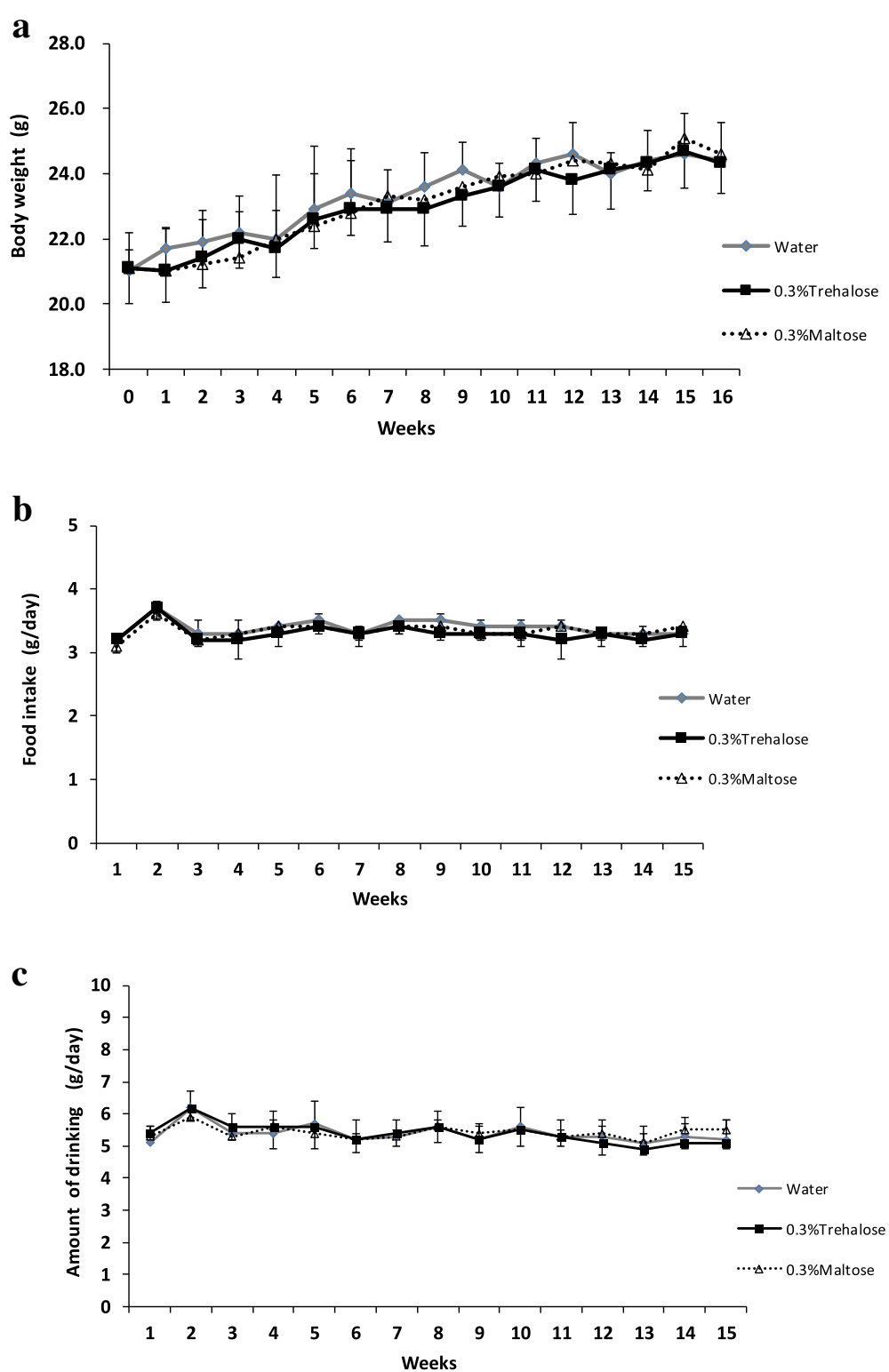

Fig. 2 The effect of drinking water containing trehalose on body weight, food intake, and water consumption. The body weight (a), food intake (b) and water consumption (c) per mouse were measured every week during the experiment. Values were shown as means \pm standard deviations $(n=16)$. Statistical analysis was performed using non-parametric Steel-Dwass. There was no significant difference among the three groups in the respective parameters $(p<0.05)$

Table 2 Body, adipose tissue and organ weights in mice after 16 weeks of trehalose intake

\begin{tabular}{|c|c|c|c|c|}
\hline & & Water & Trehalose & Maltose \\
\hline Body weight (g) & & $24.6 \pm 1.11$ & $24.4 \pm 1.20$ & $24.3 \pm 1.22$ \\
\hline \multirow[t]{3}{*}{ Adipose weight (g) } & Mesenteric adipose & $0.23 \pm 0.05$ & $0.23 \pm 0.04$ & $0.21 \pm 0.05$ \\
\hline & Perirenal adipose & $0.15 \pm 0.06$ & $0.15 \pm 0.05$ & $0.14 \pm 0.04$ \\
\hline & Retroperitoneal adipose & $0.34 \pm 0.11$ & $0.35 \pm 0.12$ & $0.31 \pm 0.10$ \\
\hline Liver (g) & & $1.28 \pm 0.09$ & $1.27 \pm 0.15$ & $1.24 \pm 0.07$ \\
\hline
\end{tabular}

Values are means \pm standard deviation of 8 mice per group. There are no significant differences between the 3 groups $(p<0.05)$. Statistical analysis was performed by non-parametric Steel-Dwass 

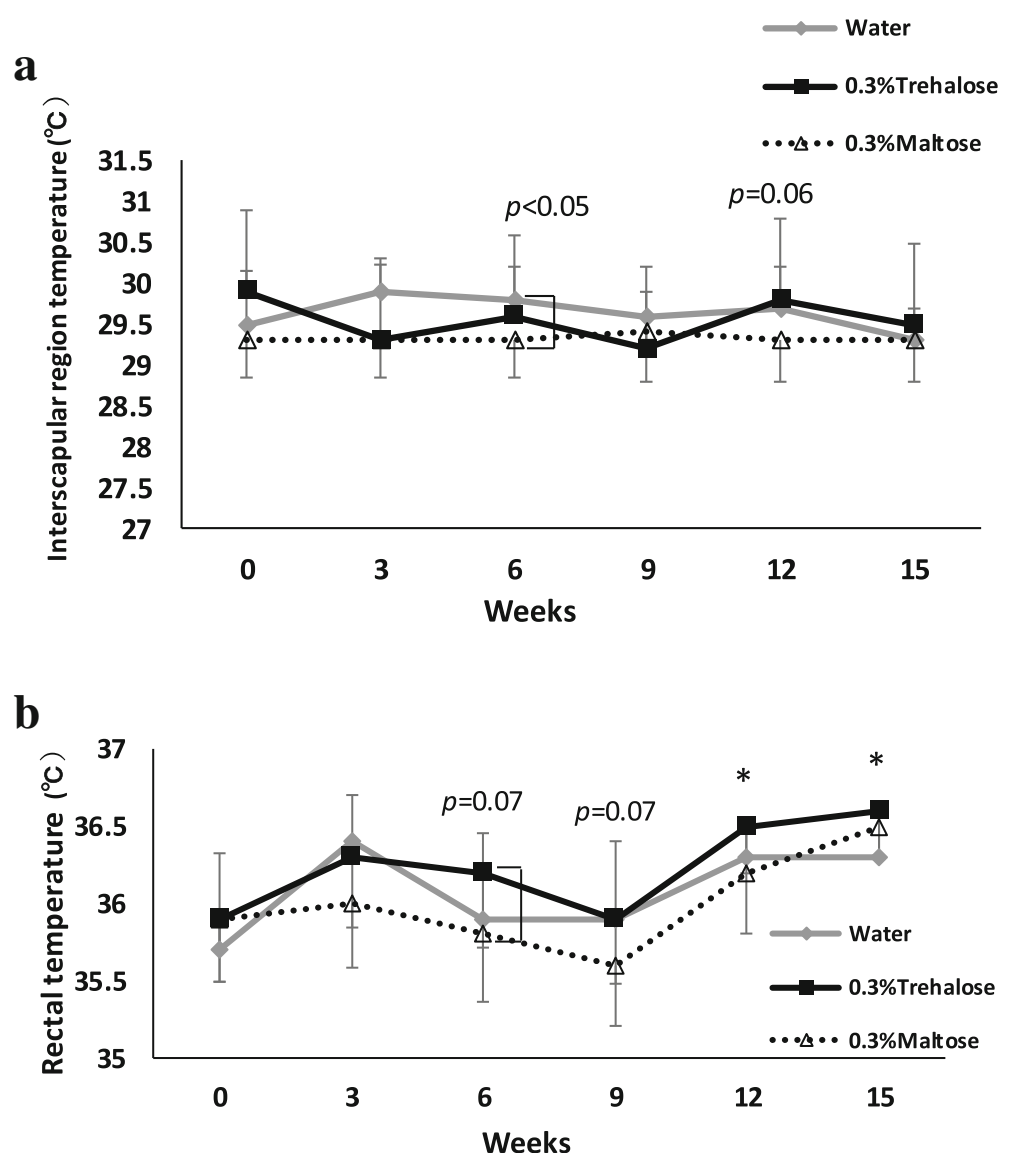

Fig. 3 The effect of drinking water containing trehalose on body temperature. Every 3 weeks, interscapular (a) and rectal temperature (b) were measured. Values were shown as means \pm standard deviations $(n=16)$. Statistical analysis was performed using Tukey-Kramer and Steel-Dwass. Interscapular temperature (a): Values show statistical significance at 6 weeks of the experiment (Water vs Maltose; $p<0.05)$. Rectal temperature $(\mathbf{b})$ : values show statistical significance at 12 weeks (Trehalose vs Maltose; $p<0.05$ ) and at 15 weeks of the experiments (Trehalose vs Water; $p<0.05$ )

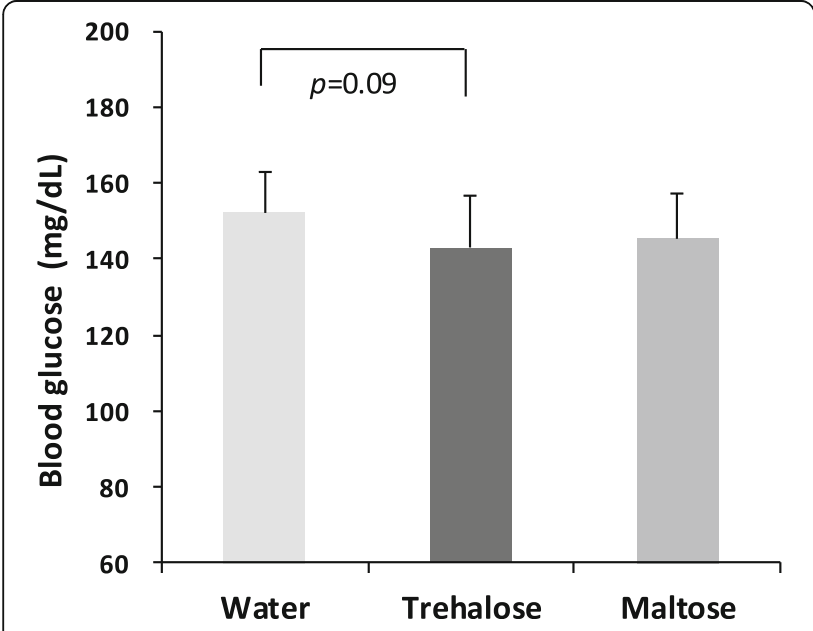

Fig. 4 The effect of drinking water containing trehalose on blood glucose levels. Non-fasting blood glucose was measured after a light phase of 2 to $3.5 \mathrm{~h}$ at 15 weeks of the experiment. Values are shown as means \pm standard deviations $(n=16)$. Statistical analysis was performed using Tukey-Kramer water and the maltose groups consisted of many large uniocular white adipocytes, whereas the WAT in the trehalose group consisted of many multilocular adipocytes with small lipid droplets (Fig. 5a). Surprisingly, even under the normal dietary conditions, the size of the mesenteric adipocytes in the trehalose group $\left(732 \pm 70 \mu \mathrm{m}^{2}\right)$ was significantly smaller than those of the water $\left(1094 \pm 212 \mu \mathrm{m}^{2}\right)$ and the maltose groups $\left(1138 \pm 218 \mu \mathrm{m}^{2}\right) \quad(p<0.01) \quad$ (Fig. $\left.5 \mathrm{~b}\right)$. Moreover, the size of the subcutaneous inguinal adipocytes was also smaller in the trehalose $(738 \pm$ $\left.184 \mu \mathrm{m}^{2}\right)$ group than in the water $\left(1412 \pm 244 \mu \mathrm{m}^{2}\right)$ and the maltose groups $\left(1155 \pm 136 \mu \mathrm{m}^{2}\right)(p<0.01)$, as well as in the case of the mesenteric adipocytes (Fig. 5c). In both the mesenteric and inguinal adipose tissues, the peak of the frequencies of adipocyte size in the trehalose group was less than $400 \mu \mathrm{m}^{2}$, whereas those of the water and maltose groups were from 1500 to $2000 \mu \mathrm{m}^{2}$ (Fig. 5d and e). 

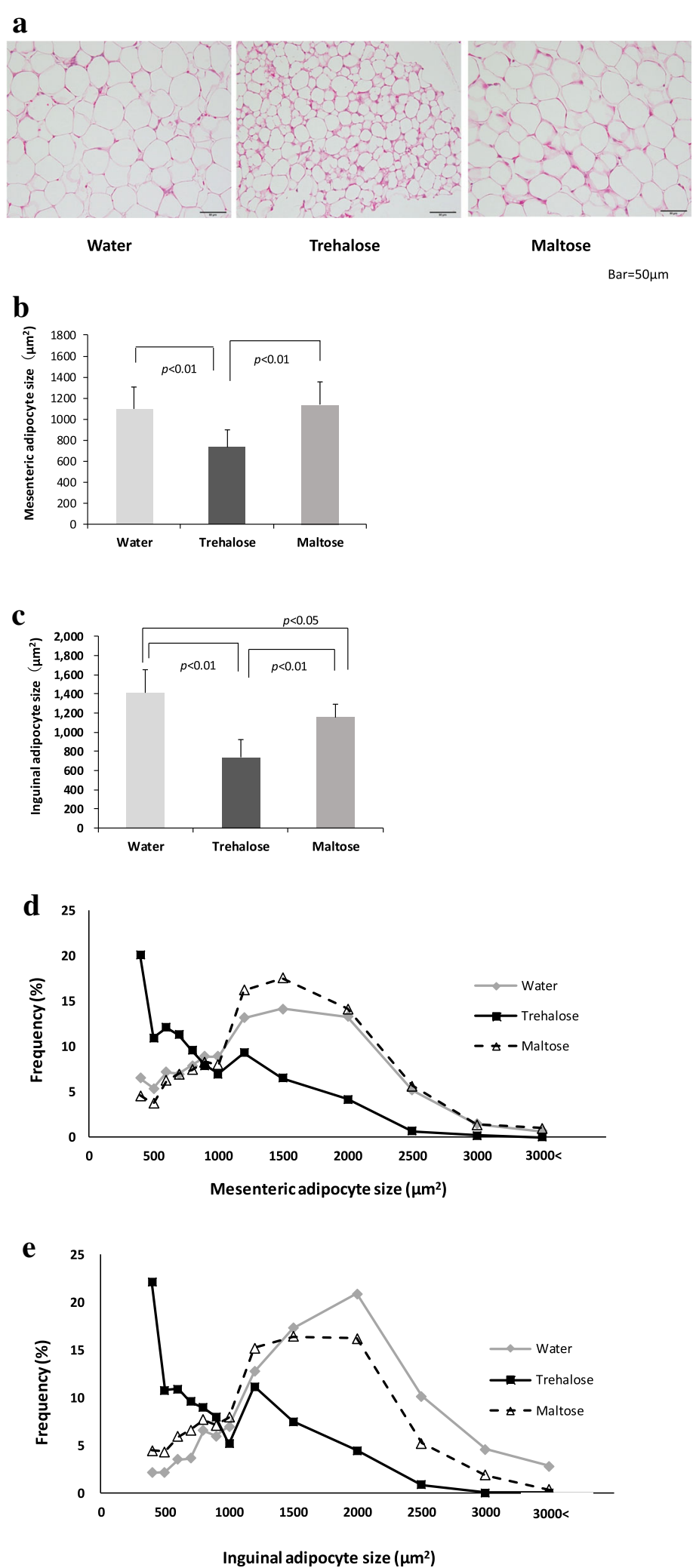

Fig. 5 (See legend on next page.) 
(See figure on previous page.)

Fig. 5 The effect of drinking water containing trehalose on adipocyte size in mesenteric and inguinal adipose tissue. The histology of WAT in the mesenteric and the inguinal adipose tissues and also the adipocyte size of both WATs were measured using the image software cellSens. Representative images of hematoxylin-eosin staining in sections of inguinal adipose tissue $(\times 400)$ are shown to assess histologically (a) and determine the size of mesenteric adipocytes (b) and inguinal adipocytes (c). In addition, cell size profiling in mesenteric adipose tissue (d) and inguinal adipose tissue $(\mathbf{e})$ is summarized. Values are shown as means \pm standard deviations $(n=8)$. Statistical analysis was performed with TukeyKramer. Values show statistical significance ( $5 \mathrm{~b}$ and $5 c ; p<0.01,5 c ; p<0.05)$. WAT: white adipose tissue

Trehalose increased the proportion of UCP1 positive cells in mesenteric and inguinal adipose tissues

Next, we examined the numbers of beige cells contained in these adipose tissues. UCP1 staining was used to identify the beige cells in mesenteric and inguinal adipose tissues
(Fig. 6a). As shown in Fig. 6a, UCP1 positive cells were stained blue in their cytoplasm. In the trehalose group, WAT was interspersed with a large number of multilocular cells, but those cells were scarce in WAT from the water and the maltose groups. The numbers of UCP1 positive cells

a

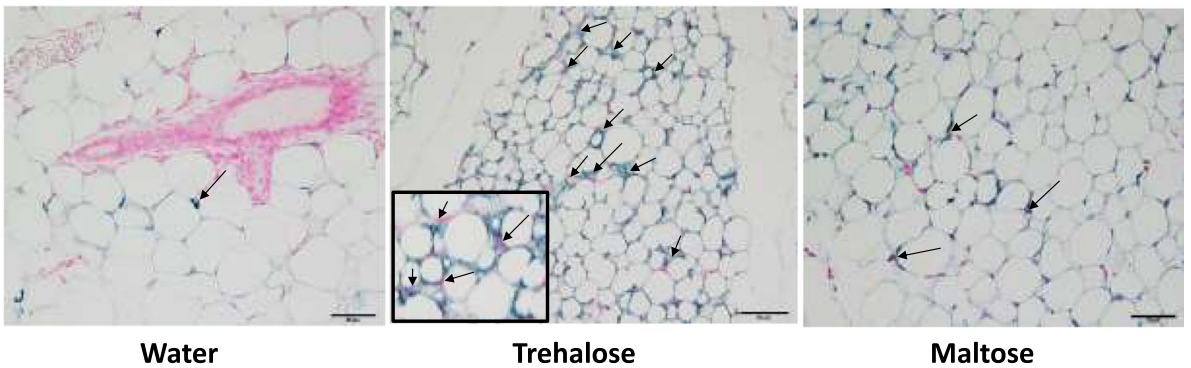

Bar $=50 \mu \mathrm{m}$

b
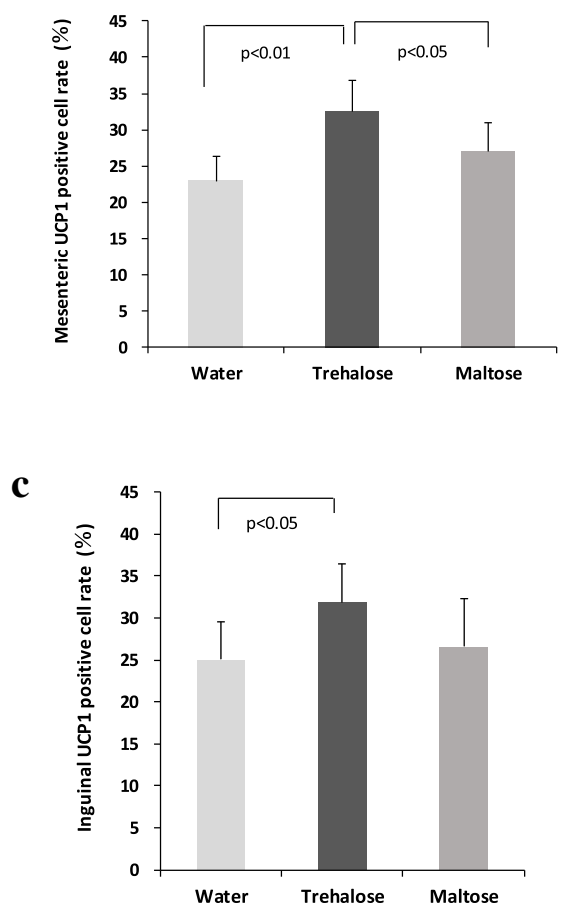

Fig. 6 The effect of drinking water containing trehalose on browning in mesenteric and inguinal adipose tissue. UCP1 positive cells were stained blue with anti-UCP1 antibody $(\times 400)$ in the respective pictures $(\mathbf{a})$. Arrows $(\rightarrow)$ indicate UCP1 positive cells. The inset is a magnified image of UCP1 positive cells $(\times 1000)$. The proportion of UCP1 positive cells in mesenteric adipose tissue $(\mathbf{b})$. The proportion of UCP1 positive cells in inguinal adipose tissue $(\mathbf{c})$. Values are shown as means \pm standard deviations $(n=8)$. Statistical analysis was performed using Tukey-Kramer. Values show statistical significance $(6 \mathrm{~b} ; p<0.01,6 \mathrm{~b}$ and $6 c ; p<0.05)$. UCP1: uncoupling protein 1 
were counted. The rates of the UCP1 positive cells in the total adipocytes are shown in Fig. 6b and c. In the mesenteric adipose tissue, a significantly higher rate of UCP1 positive cells was found in the trehalose group $(32.6 \pm 4.2 \%)$ than in the water $(23.0 \pm 3.4 \%)(p<0.01)$ and the maltose groups $(27.1 \pm 3.9 \%)(p<0.05)$ (Fig. $6 \mathrm{~b})$. Similarly, in the inguinal adipose tissue, the rate of UCP1 positive cells was significantly higher in the trehalose group $(31.8 \pm 4.6 \%)$ than in the water group $(25.1 \pm 4.5 \%)(p<0.05)$, but that of the maltose group was not statistically different (Fig. 6c).

\section{Increased rates of UCP1 positive cells correlated with decreased adipocyte size}

These results suggest that trehalose induced UCP1 positive beige cells in relation to the suppression of adipocyte hypertrophy. To confirm the relationship, therefore, we examined the correlation between adipocyte size and UCP1 positive ratio ( $n=8$, each group). In both the mesenteric and the inguinal adipose tissues, there was a negative correlation between the adipocyte size and the rates of UCP1 positive cells, and their correlation coefficients were $\mathrm{r}=-0.55(p<0.01)$ and $\mathrm{r}=-0.60(p<0.01)$, respectively (Fig. 7a and b). When more UCP1 positive cells were induced in the adipose tissues, it was found that the white adipocytes became smaller in size.

\section{Trehalose induced expression of beige adipocyte-related genes}

Furthermore, we measured the expression of genes associated with beige adipocytes. According to the previous paper [6, 23, 24], the four genes Cidea (cell deathinducing DNA fragmentation factor- $\alpha$-like effector A), Ucp1, Prdm16 (PR domain containing 16), and Pgc1 $\alpha$ (PPAR $\gamma$ coactivator $1 \alpha$ ) were selected as markers for beige adipocytes. Cidea is a gene that encodes a lipid droplet protein to regulate triglyceride deposition in adipocytes. Prdm16 encodes a zinc finger protein and functions as a transcriptional coregulator to control the development of brown adipocytes. Pgc1 $\alpha$ is a gene encoding a transcriptional coactivator and is defined as a master regulator of mitochondrial biogenesis. In the mesenteric adipose tissue, mRNA expression levels of Cidea, Prdm16, and Pgcla in the trehalose group showed a tendency to be higher than those in the water and the maltose groups, although there were no significant differences (Fig. 8a). In the inguinal adipose tissue, the cidea expression level in the trehalose group was significantly higher compared to those of the water and the maltose groups $(p<0.05)$. Among the other genes, the Ucp1 expression level in the trehalose group was significantly higher than that in the maltose group $(p<0.05)$, but not in the water group (Fig. 8b).

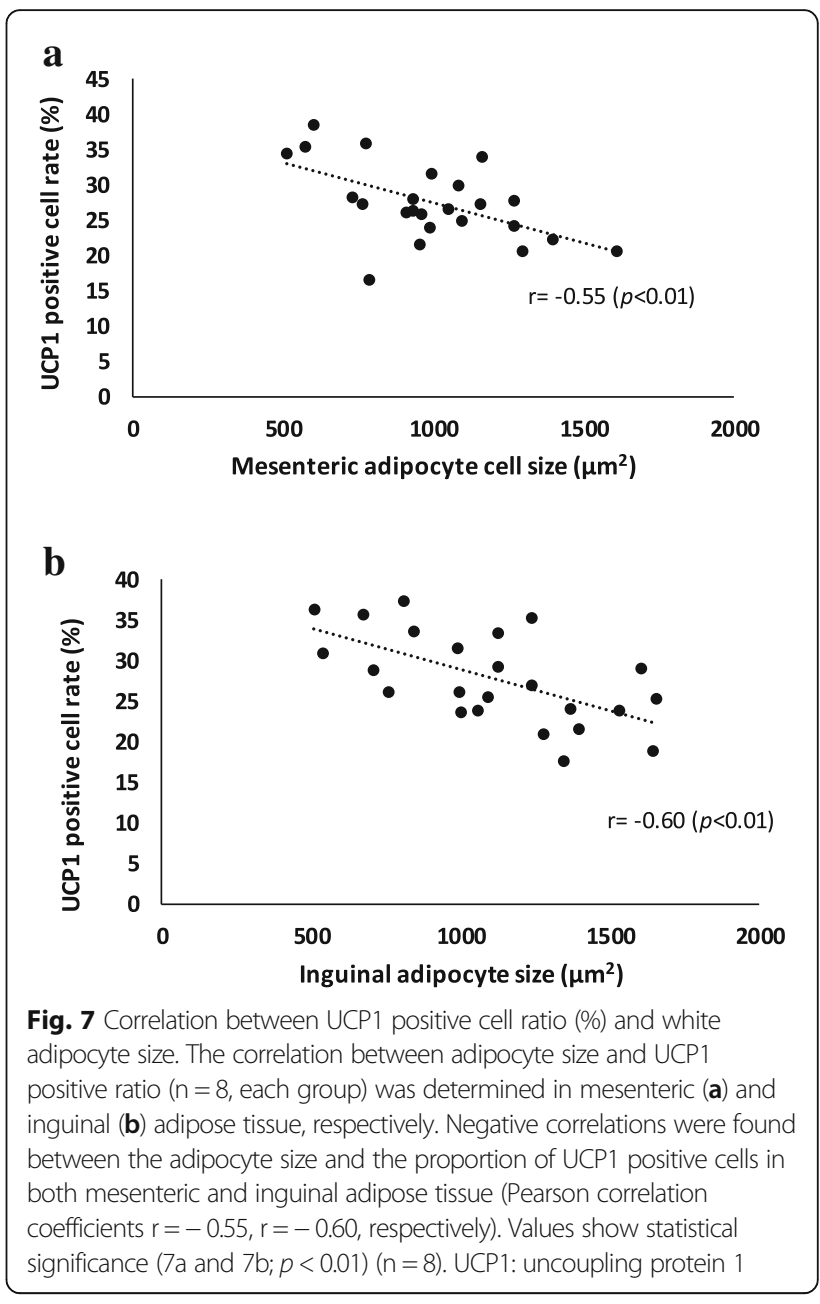

Trehalose did not induce more severe fatty degeneration than maltose

We examined whether trehalose administration affected BAT, a representative heat-producing tissue. Since fatty degeneration of BAT was observed in the saccharide (trehalose or maltose)-administered groups, as shown in Fig. 9a, we compared the severity among the three groups by the histopathological method of fatty degeneration scores. In the water group, the BAT of all mice showed a normal structure and their fatty degeneration scores were zero. In contrast, both the trehalose and the maltose groups exhibited higher and varying degrees of fatty degeneration scores compared with those of the water group. However, the fatty degeneration score of the trehalose group was significantly lower than that of the maltose group $(p<0.05)$ (Fig. 9b).

\section{Trehalose had no effect on serum levels of FGF-21}

FGF-21 is a circulating hepatokine that is a potential drug to regulate extrahepatic energy homeostasis and induce BAT activation or WAT browning. Therefore, serum FGF-21 levels were measured to investigate the 

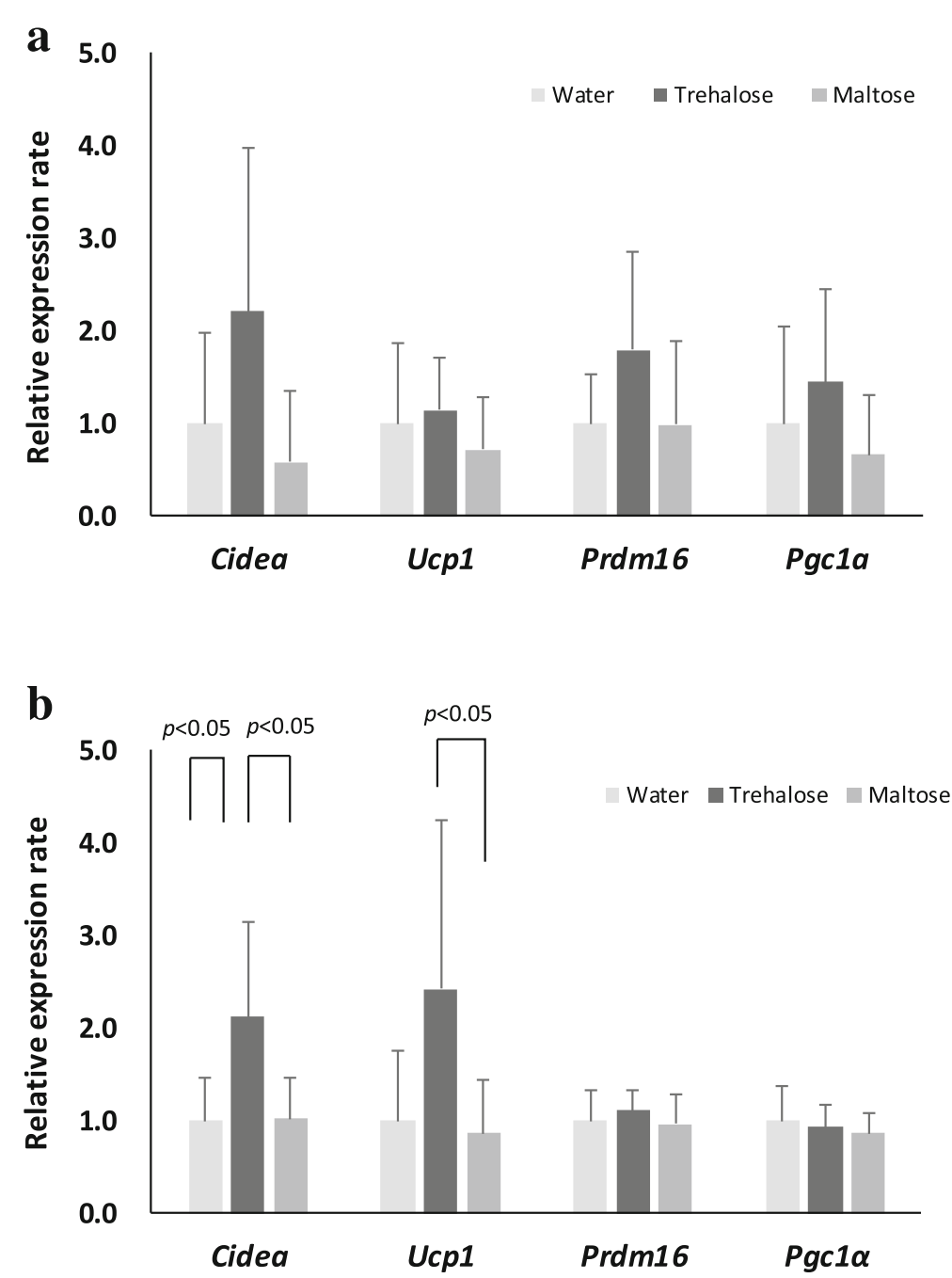

Fig. 8 The effect of drinking water containing trehalose on beige-adipocyte-related gene expression. Total RNAs were extracted from mesenteric adipose tissues and inguinal adipose tissues, and the respective CDNAs were prepared as described in the manual. Subsequently, real-time PCR was performed using a LightCycler 480 system. For quantitative purposes, expression levels of the respective target genes were normalized to the housekeeping gene 18S rRNA. Mesenteric adipose tissues (a) and inguinal adipose tissues (b) are shown. Values are shown as means \pm standard deviations $(n=8)$. Cidea expression was significantly higher in the trehalose group compared with both the water and the maltose groups (8b; $p<0.05$, Steel). Ucp1 expression was significantly higher in the trehalose group compared with the maltose group (8b; $p<0.05$, Tukey-Kramer). Cidea: cell death-inducing DNA fragmentation factor-a-like effector A; UCP1: uncoupling protein 1

possible involvement of FGF-21 in trehalose-induced browning. As shown in Fig. 10, the serum levels of FGF21 were not different among the three groups.

\section{Discussion}

In the present study, we demonstrated that drinking water containing trehalose with a normal diet significantly induced WAT browning in inguinal and mesenteric adipose tissues, with significant upregulation of the beige adipocyte-related genes Cidea and Ucp1. To our knowledge, this is the first report to evaluate the in vivo effects of disaccharide on WAT browning by immunohistochemical methods. We deduced that these effects resulted in elevated body temperature in the rectal region. Moreover, we found a strong correlation between the increase in the rate of UCP1 positive cells and the decrease in adipocyte hypertrophy, indicating that WAT browning is involved in the mechanism for the regulation of adipocyte size.

Regarding the UCP1 expression, there was a discrepancy between the gene and the protein expression levels in the mesenteric tissue, but this contradiction was not observed in the inguinal adipose tissue. The reasons are as follows: 1) samples from the mesenteric preparation may contain more non-adipose tissue than those from the inguinal preparation and 2) Ucp1 gene expression is 


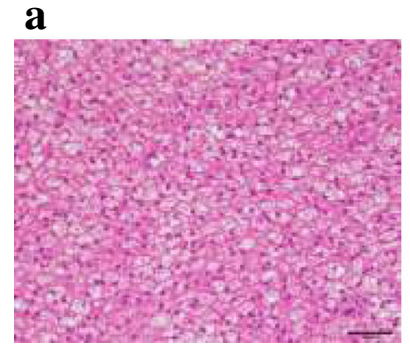

Water

b

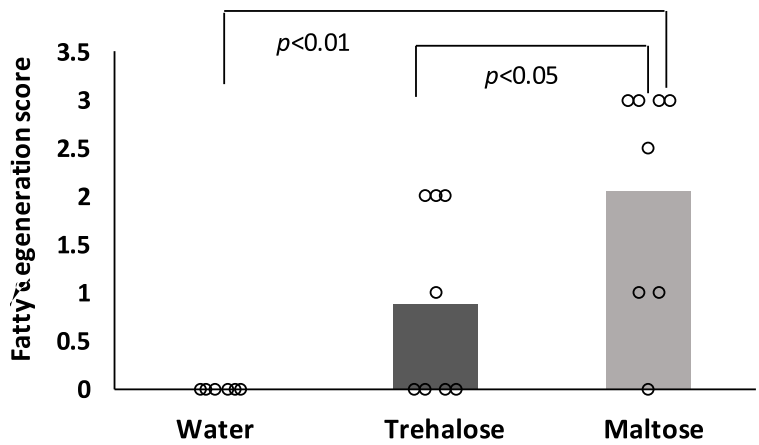

Fig. 9 Effect of drinking water containing trehalose on BAT. Histological analysis of BAT (a) hematoxylin-eosin staining, $\times 400)$. Arrows $(\rightarrow)$ indicate vacuolation of BAT cells by fat deposition. Fatty degeneration scores (b) were graded as negative: 0, slightly: 1, mildly: 2, and severe: 3 . Subsequently, the personal score was plotted $(n=8)$. Statistical analysis was performed using the nonparametric Wilcoxon test. Values show statistical significance (9b; $p<0.01$ and $p<0.05)$. BAT: brown adipose tissue

more inducible in the inguinal adipose tissue than in the mesenteric adipose tissue $[25,26]$.

It is well known that there are three possible ways to induce thermogenesis: 1) increase of BAT activity in existing classical brown adipocytes, 2) induction of formation of

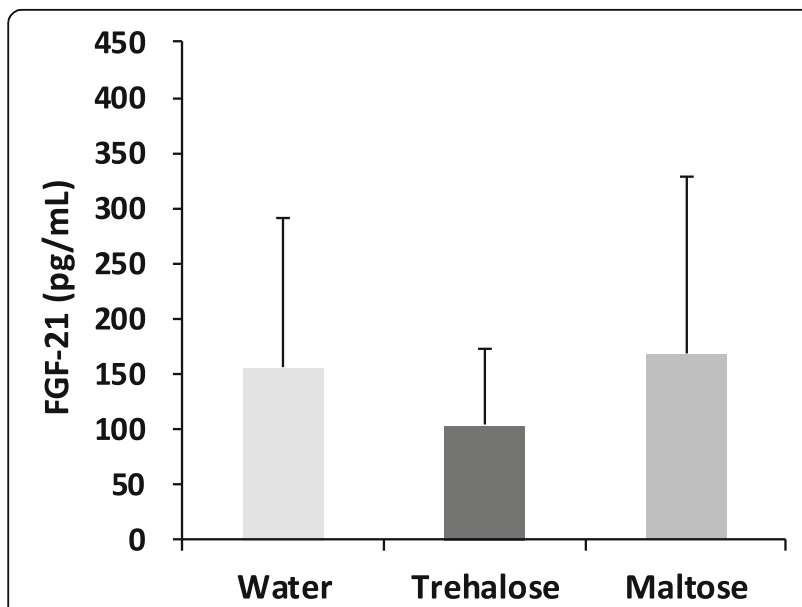

Fig. 10 Effect of drinking water containing trehalose on FGF-21 protein levels in serum. Serum FGF-21 protein was measured at the end of the experiment. Values were shown as means \pm standard deviations $(n=8)$. Statistical analysis was performed using nonparametric Steel-Dwass. FGF-21: fibroblast growth factor-21 new adipocytes from adipogenic progenitor cells, and 3) switching of existing white adipocytes to beige adipocytes in subcutaneous fat (WAT browning). We provided evidence that trehalose exerts thermogenic activity through the WAT browning mechanism. It is unlikely that trehalose promoted BAT activity, as the histological analysis of BAT did not show such evidence in our experiments. Although the second possibility of progenitor cell differentiation is quite interesting, further investigation is required to verify the possibility.

In general, thermogenic stimulation such as cold exposure exerts its effects by stimulating the sympathetic nervous system (SNS) to release norepinephrine and then increasing the thermogenesis of BAT through $\beta 3$ adrenergic receptor (adrb3) activation. Several dietary components have been described as thermogenic molecules exhibiting browning ability, including capsaicin, resveratrol, curcumin, green tea, berberine, and fish oil [27, 28]. Capsaicin and fish oil exert thermogenic effects by indirect activation of the adrb3, which is first induced by their binding to transient receptor potential vanilloid 1 and is followed by stimulation of SNS and release of norepinephrine. Curcumin and green tea have been shown to be effective in inducing WAT browning and thermogenesis through the mechanism of mitochondrial 
biogenesis and adrb3 activation. Since trehalose could directly induce browning in adipocytes in cell culture (data not shown), we deduced that the action of trehalose was not mediated by the SNS. It has also been suggested that resveratrol increases thermogenesis by stimulating mitochondrial biogenesis and increasing beige adipocytes. The underlying mechanism is thought to involve the activation of AMP-activated protein kinase (AMPK) and its downstream pathway to nicotinamide adenine dinucleotide (NAD)-sirtuin1-PCG1 $\alpha$ [23, 29]. Furthermore, berberine, an alkaloid compound found in various herbs, has been shown to regulate adaptive thermogenesis, and that mechanism may involve AMPK and Pgcl $\alpha$ activation. Since we have previously demonstrated that trehalose increases serum high molecular weight adiponectin and mitigates insulin resistance in HFD-fed mice [19], on the other hand, the possibility to activate AMPK-Pgc1 $\alpha$ downstream of adiponectin was also considered. As another mechanism, berberine has been reported to decrease inflammatory markers such as TNF- $\alpha$ and IL-6, and those anti-inflammatory effects are presumed to enhance WAT browning by M2 macrophage polarization [30]. Since trehalose could also exert antiinflammatory effects, we predicted these effects in WAT browning. However, histological observations in our studies did not show significant infiltration of eosinophils and M2 macrophages in WAT.

Recently, Zang et al. [31] demonstrated that trehalose induces thermogenesis in a hepatocyte transcription factor EB (TFEB)-dependent manner, with a concomitant upregulation of Ucp1 expression in hepatic and white adipose tissue. They proposed a mechanism in which trehalose first induces the hepatic fasting response by inhibiting the glucose transporter hepatocyte solute carrier 2A (SLC2A), and subsequently triggers AMPK$P g c 1 \alpha$-TFEB-FGF-21 signaling. In this mechanism, hepatocytes are an important part of the action of trehalose, and systemic thermogenesis is supposed to be partially induced by the hepatocyte-centered fasting-like response. Therefore, we measured FGF-21 protein, which plays a central role in systemic thermogenesis. However, the serum FGF-21 protein was not elevated by trehalose administration in comparison to the water or the maltose groups. We do not know the exact reason for this discrepancy with the results of Zang et al. [31], but we assume that differences in the experimental conditions caused the inconsistency. In their experiments, 3\% (W/ V) trehalose was administered with drinking water for 5 days, while we administered $0.3 \%(\mathrm{~W} / \mathrm{V})$ trehalose for 16 weeks. That is, their administration was conducted with higher concentrations and for shorter periods of time compared with ours. Another possibility has been proposed by Fisher et al. [32]. They demonstrated that cold exposure induces browning in WAT without increasing the circulating FGF-21 levels, suggesting that upregulation of FGF-21 gene expression itself in WAT can operate thermogenesis signaling. However, it has not yet been possible to prove the involvement of FGF-21 in the mechanism of WAT browning by trehalose administration. Otherwise, our results may suggest the presence of distinct signal pathways.

On the other hand, Chevalier et al. [33] demonstrated that cold exposure leads to marked changes in the gut microbiota, and this "cold microbiota" phenomenon increases WAT browning. Therefore, we think that changes in the microbiota may be one of the browning mechanisms of trehalose. We are now planning some experiments to test our hypothesis.

\section{Conclusions}

We have demonstrated that drinking water containing trehalose induced WAT browning accompanied with suppression of white adipocyte hypertrophy, elevation of body temperature and mitigation of blood glucose levels, even under normal diet conditions and at a lower concentration compared with previous studies. Therefore, we propose trehalose as a new type of thermogenic dietary component for improving human health by preventing obesity and promoting WAT browning.

\section{Abbreviations \\ adrb3: beta-3 adrenergic receptor; AMPK: AMP-activated protein kinase; BAT: Brown adipose tissue; Cidea: Cell death-inducing DNA fragmentation factor- a-like effector A; FGF-21: Fibroblast growth factor-21; HFD: High fat diet; IL- 6: Interleukin-6; NAD: Nicotinamide adenine dinucleotide; PAI-1: Plasminogen activator inhibitor-1; PCR: Polymerase chain reaction; Pgcla: PPARy coactivator 1a; Prdm 16: PR domain containing 16; SLC2A: Solute carrier 2A; SNS: Sympathetic nervous system; TFEB: Transcription factor EB; TNF-a: Tumor necrosis factor-a; UCP1: Uncoupling protein 1; VN: Volume/volume; WN: Weight/volume; WAT: White adipose tissue}

\section{Acknowledgements}

We thank Dr. Shuji Nakamura in our company for reviewing the manuscript.

\begin{abstract}
Authors' contributions
Design of the study was done by CA, NA, SA, CY, SE and HM. Experiments were performed by CA, NA, SA, CY, SM, AM and AS. Analysis and data mining were performed by CA, NA and SA. Technical assistance was performed by SA, CY, SM, AM and AS. Interpretation of the information obtained was done by NA, SE, TA, HM and CA. The manuscript was written by CA, NA and approved by HM, TA and US. All authors have read and approved the final manuscript.
\end{abstract}

\section{Funding}

Not applicable.

\section{Availability of data and materials Not applicable.}

Ethics approval and consent to participate

All experimental procedures were conducted in accordance with the Guidelines for Care and Use of Laboratory Animals of the Hayashibara Co., Ltd.

Consent for publication

All authors support the submission to this journal. 


\section{Competing interests}

All authors are employees of Hayashibara Co. Ltd., and all funds for the study were sponsored. The authors declare that they have no other conflicts of interest.

Received: 16 April 2019 Accepted: 26 June 2019

Published online: 16 July 2019

\section{References}

1. Guilherme A, Virbasius JV, Puri V, Czech MP. Adipocyte dysfunctions linking obesity to insulin resistance and type 2 diabetes. Nat Rev Mol Cell Biol. 2008;9:367-77.

2. Coelho M, Oliveira T, Fernandes R. Biochemistry of adipose tissue: an endocrine organ. Arch Med Sci. 2013;9:191-200.

3. Saito M. Brown adipocyte tissue as a regulator of energy expenditure and body fat in humans. Diabetes Metab J. 2013;37:22-9.

4. Wang W, Seale P. Control of brown and beige fat development. Nat Rev Mol Cell Biol. 2016;17:691-702.

5. Vargas-Castillo A, Fuentes-Romero R, Rodriguez-Lopez LA, Torres N, Tovar AR. Understanding the biology of thermogenic fat: is browning a new approach to the treatment of obesity? Arch Med Res. 2017;48:401-13.

6. Sidossis L, Kajimura S. Brown and beige fat in humans: thermogenic adipocytes that control energy and glucose homeostasis. J Clin Invest. 2015; 125:478-86.

7. Virtanen KA, Lidell ME, Orava J, Heglind M, Westergren R, Niemi T, Taittonen M, Laine J, Savisto NJ, Enerbäck S, Nuutila P. Functional brown adipose tissue in healthy adults. N Engl J Med. 2009;360:1518-25.

8. Wu J, Boström P, Sparks LM, Ye L, Choi JH, Giang AH, Khandekar M, Virtanen KA, Nuutila P, Schaart G, Huang K, Tu H, van Marken Lichtenbelt WD, Hoeks J, Enerbäck S, Schrauwen P, Spiegelman BM. Beige adipocytes are a distinct type of thermogenic fat cell in mouse and human. Cell. 2012;150:366-76.

9. Lizcano F, Vargas D. Biology of beige adipocyte and possible therapy for type 2 diabetes and obesity. Int J Endoclinol. 2016;2016:9542061.

10. Garcia RA, Roemmich JN, Claycombe KJ. Evaluation of markers of beige adipocytes in white adipose tissue of the mouse. Nutr Metab (Lond). 2016 13:24-37.

11. Nishizaki Y, Yoshizane C, Toshimori Y, Arai N, Akamatsu S, Hanaya T, Arai S, Ikeda M, Kurimoto M. Disaccharide-trehalose inhibits bone resorption in ovariectomized mice. Nutr Res. 2000;20:653-64.

12. Yoshizane C, Arai N, Arai C, Yamamoto M, Nishizaki Y, Hanaya T, Arai S, Ikeda M, Kurimoto M. Trehalose suppresses osteoclast differentiation in ovariectomized mice: correlation with decreased in vitro interleukin-6 production by bone marrow cells. Nutr Res. 2000;20:1485-91.

13. Arai C, Kohguchi M, Akamatsu S, Arai N, Yoshizane C, Hasegawa N, Hanaya T, Arai S, Ikeda M, Kurimoto M. Trehalose suppresses lipopolysaccharideinduced osteoclastogenesis bone marrow in mice. Nutr Res. 2001;21:993-9.

14. Sarkar S, Davies JE, Huang Z, Tunnacliffe A, Rubinsztein DC. Trehalose, a novel mTOR-independent autophagy enhancer, accelerates the clearance of mutant huntingtin and alpha-synuclein. J Biol Chem. 2007;282:5641-52.

15. Sergin I, Evans TD, Zhang X, Bhattacharya S, Stokes CJ, Song E, Ali S, Dehestani B, Holloway KB, Micevych PS, Javaheri A, Crowley JR, Ballabio A, Schilling JD, Epelman S, Weihl CC, Diwan A, Fan D, Zayed MA, Razani B. Exploiting macrophage autophagy-lysosomal biogenesis as a therapy for atherosclerosis. Nat Commun. 2017;8:15750.

16. Oku T, Nakamura S. Estimation of intestinal trehalase activity from a laxative threshold of trehalose and lactulose on healty female subjects. Eur J Clin Nutr. 2000;54:783-8.

17. Yoshizane C, Mizote A, Yamada M, Arai N, Arai S, Maruta K, Mitsuzumi H, Ariyasu T, Ushio S, Fukuda S. Glycemic, insulinemic and incretin responses after oral trehalose ingestion in healthy subjects. Nutr J. 2017;16:9.

18. Arai C, Arai N, Mizote A, Kohno K, Iwaki K, Hanaya T, Arai S, Ushio S, Fukuda S. Trehalose prevents adipocyte hypertrophy and mitigates insulin resistance. Nutr Res. 2010;30:840-8.

19. Arai C, Miyake M, Matsumoto Y, Mizote A, Yoshizane C, Hanaya Y, Koide $K$, Yamada M, Hanaya T, Arai S, Fukuda S. Trehalose prevents adipocyte hypertrophy and mitigates insulin resistance in mice with established obesity. J Nutr Sci Vitaminol (Tokyo). 2013;59:393-401.

20. Arai C, Miyata M, Yoshizane C, Koide K, Mizote A, Arai N, Hanaya T, Arai S, Fukuda S. Trehalose protect islets of Langerhans in HFD-fed obese mice: a morphometric analysis. J Jpn Soc Nutr Food Sci. 2013;66:17-24.
21. Blüher M, Michael MD, Peroni OD, Ueki K, Carter N, Kahn BB, Kahn CR. Adipose tissue selective insulin receptor knockout protects against obesity and obesity-related glucose intolerance. Dev Cell. 2002;3:25-38.

22. Mizote A, Yamada M, Yoshizane C, Arai N, Maruta K, Arai S, Endo S, Ogawa $R$, Mitsuzumi $H$, Ariyasu T, Fukuda S. Daily intake of trehalose is effective in the prevention of lifestyle-related disease in individuals with risk factors for metabolic syndrome. J Nutr Sci Vitaminol (Tokyo). 2016;62:380-7.

23. Wang S, Liang X, Yang Q, Fu X, Rogers CJ, Zhu M, Rodgers BD, Jiang Q, Dodson MV, Du M. Resveratrol induces brown-like adipocyte formation in white fat through activation of AMP-activated protein kinase (AMPK) a1. Int J Obes. 2015;39:967-76.

24. Kim M, Goto T, Yu R, Uchida K, Tominaga M, Kano Y, Takahashi N, Kawada T. Fish oil intake induces UCP1 upregulation in brown and white adipose tissue via the sympathetic nervous system. Sci Rep. 2015. https://doi.org/10.1038/srep18013.

25. Tchkonia T, Thomou T, Zhu Y, Karagiannides I, Pothoulakis C, Jensen MD, Kirkland JL. Mechanisms and metabolic implications of regional differences among fat depots. Cell Metab. 2013;17:644-56.

26. Hashimoto O, Funaba M, Sekiyama K, Doi S, Shindo D, Satoh R, Itoi H, Oiwa H, Morita M, Suzuki C, Sugiyama M, Yamakawa N, Takada H, Matsumura S, Inoue K, Oyadomari S, Sugino H, Kurisaki A. Activin E controls energy homeostasis in both brown and white adipose tissues as a hepatokine. Cell Rep. 2018;25:1193-203.

27. Okla M, Kim J, Koehler K, Chung S. Dietary factors promoting brown and beige fat development and thermogenesis. Adv Nutr. 2017;8:473-83.

28. Silvester AJ, Aseer KR, Yun JW. Dietary polyphenols and their roles in fat browning. J Nutr Biochem. 2019;64:1-12.

29. Zou T, Chen D, Yang Q, Wang B, Zhu MJ, Nathanielsz PW, Du M. Resveratrol supplementation of high-fat diet-fed pregnant mice promotes brown and beige adipocyte development and prevents obesity in male offspring. J Physiol. 2017;595:1547-62.

30. Fabbiano S, Suárez-Zamorano N, Rigo D, Veyrat-Durebex C, Stevanovic Dokic A, Colin DJ, Trajkovski M. Coloric restriction leads to browning of white adipose tissue through type 2 immune signaling. Cell Metab. 2016;24:434-46.

31. Zhang Y, Higgins CB, Mayer AL, Mysorekar IU, Razani B, Graham MJ, Hruz PW, DeBosch BJ. TFEB-dependent induction of thermogenesis by the hepatocyte SLC2A inhibitor trehalose. Autophagy. 2018;14:1959-75.

32. Fisher FM, Kleiner S, Douris N, Fox EC, Mepani RJ, Verdeguer F, Wu J, Kharitonenkov A, Flier JS, Maratos-Flier E, Spiegelman BM. FGF21 regulates PGC-1a and browning of white adipose tissues in adaptive thermogenesis. Genes Dev. 2012;26:271-81.

33. Chevalier C, Stojanović O, Colin DJ, Suarez-Zamorano N, Tarallo V, VeyratDurebex C, Rigo D, Fabbiano S, Stevanović A, Hagemann S, Montet X, Seimbille Y, Zamboni N, Hapfelmeier S, Trajkovski M. Gut microbiota orchestrates energy homeostasis during cold. Cell. 2015;163:1360-74.

\section{Publisher's Note}

Springer Nature remains neutral with regard to jurisdictional claims in published maps and institutional affiliations.
Ready to submit your research? Choose BMC and benefit from:
- fast, convenient online submission
- thorough peer review by experienced researchers in your field
- rapid publication on acceptance
- support for research data, including large and complex data types
- gold Open Access which fosters wider collaboration and increased citations
- maximum visibility for your research: over $100 \mathrm{M}$ website views per year
At BMC, research is always in progress. 